\title{
SOME ASPECTS OF THE MATERIALS' OPTICAL LIMITING FEATURES
} Natalia Kamanina ${ }^{1}$

\begin{abstract}
Due to the effective use of the fullerenes and other nanoparticles in the materials for the general optoelectronic, laser and display technique, as well as for biomedicine, the different mechanisms to attenuate the light intensity are considered with good advantage in order to protect the human eyes and technical devises from high laser irradiation. Reverse saturation absorption, complex formation, scattering, etc. are taken into account. In the current paper the influence of the content of the nanoobjects on the interface relief is shown and considered as an additional possible optical limiting mechanism. Moreover, the diffraction from the nanostructured materials via high frequency Kerr effect is added to extend the numbers of the optical limiting mechanisms. Different experimental instruments and supporting models are presented. VIS lasers, AFM, OCA devises are applied to test and visualize the results. Theoretical and experimental data are in good coincidence.
\end{abstract}

UDC Classification: 532.783; 535; 539.6, DOI: https://doi.org/10.12955/pns.v1.118

Keywords: Organic materials, surface relief, sensitization, fullerenes and nanotubes, wetting angle, reflection and refraction

\section{Introduction}

The problem of limiting of the laser radiation of high intensity (or energy density in the pulsed mode) is one of the first places in the design of the complex optoelectronic circuits and the technical devices used in eye microsurgery and other biomedical applications. This is due to the necessary protection of the service personnel and sensitive photo electronic converters from laser breakdown. Thus, it becomes necessary to solve the problem of searching and modifying the properties of new sensitized materials, as well as to study the mechanisms responsible for limiting the radiation passing through such types of the materials.

Due to unique properties of the fullerenes (Krätschmer,1990; Krätschmer, 1990; Liu, 1994) and carbon nanotubes (Robertson, 2004; Namilae, 2004; Ould-Moussa, 2013), these sensitizers are occupying the special place in the area of the materials parameters optimization, including the features to attenuate the laser beam. Moreover, the $\pi$-conjugated organic materials (such as pyridines, polyvinyl carbazole, polysilanes, poly(3-hexylthiophene), polyimide, liquid crystal matrixes, etc.) sensitized with the mentioned above nanoobjects are the promising compounds for these purposes (Brabec, 1999; Sapurina, 2000; Kamanina, 2001; Kamanina, 2002) because they allow their physicalchemical characteristics to be modified by doping with a varied amount of these impurities.

Among different mechanisms, the optical limiting (OL) effect, a reverse saturable absorption (Couris, 1995; Belousov, 1997), intermolecular charge transfer complex formation (Hosoda, 1997; Kamanina, 2005), light-induced change of the refractive index (Kamanina, 2008; Kamanina, 2012), scattering (Belousov, 1999; Belousova, 2001), etc. can be considered.

In the current paper the novel approach to explain the OL mechanism is proposed. It is based on the nanoobjects varied content influence on the surface relief, thus, on the additional reflection from the surface. Moreover, the novel results are shown to support the OL mechanism via high-frequency Kerr effects for the sensitized liquid crystal matrix with the fullerene-containing polyaniline via energy losses due to diffraction.

\section{Experimental conditions, materials and results}

The possible model on how the content of the nanoobjects influences the interface relief is shown in Fig.1 via checking the wetting angle at the material surface. The organics matrix can be presented as the polyimides, polyvinyl alcohol (PVA), etc. compounds. It has been shown before that the change of the nanoobjects (fullerene) content in the polyimide matrix from 0.5 to $1.0 \mathrm{wt} . \%$ permitted to increase of the wetting angle up to 72 and 103 degrees respectively; when the content of the carbon nanotubes (CNTs) in the PVA matrix is $1.0 \mathrm{wt} \%$, the change of the wetting angle can be changed from 40 to 82 degrees (Kamanina, 2019). This content of the CNTs in the PVA can be increase up to $5.0 \mathrm{wt}$. before the saturation, that provokes the change of the wetting angle at the PVA surface up to 28-130 degrees. As a result, the scattering from these modified surfaces should be taken into account under the condition of the OL effect explanation.

\footnotetext{
${ }^{1}$ Vavilov State Optical Institute, St.- Petersburg, Russia; St.-Petersburg Electrotechnical University (“LETI”), St.-Petersburg, Russia, nvkamanina@mail.ru
} 
presented in Fig.2. The pulsed nanosecond Nd-laser with the second harmonic generation was used as the main irradiation source. One can testify that the light beams create the interference pictures at the organic thin films structured with nanoobjects, but this interference pattern diffracts at the nanostructured materials body. As the results, the energy can be distributed at 10 or more diffracted orders, if the $\Lambda^{-1}$ is more than the thickness of the thin films. Here $\Lambda$ is the spatial frequency and $\Lambda^{-1}$ is the period of the recorded grating. Thus, the energy losses via diffraction can be taken account to explain the attenuation of the laser beam via the OL effect.

Some results of the laser induced refractive index that change in the sensitized organic media are shown in Table 1. The data is comparatively presented for the pure LC, LC doped with the complex of polyimide $+\mathrm{C}_{70}$ and the $\mathrm{LC}$ doped with the complex PANI- $\mathrm{C}_{60}$.

Table 1: Comparative laser-induced change of the refractive index $\Delta n_{i}$ in the liquid crystal thin films doped with the complex: PANI- $\mathrm{C}_{60}$.

\begin{tabular}{|c|c|c|c|c|c|}
\hline Materials & $\begin{array}{c}\text { Content of the } \\
\text { sensitizer wt.\% }\end{array}$ & $\begin{array}{c}W, \\
\mathrm{~J} \times \mathrm{cm}^{-2}\end{array}$ & $\begin{array}{c}\tau, \\
\mathrm{ns}\end{array}$ & $\Delta n_{\mathrm{i}}$ & Ref. \\
\hline LC & 0.0 & 0.2 & & $0.16 \times 10^{-3}$ & Khoo, 1994 \\
\hline $\begin{array}{c}\text { LC doped with } \\
\text { polyimide+C }\end{array}$ & 0.2 & $0.1-0.3$ & 20 & $1.2 \times 10^{-3}$ & Kamanina, 2013 \\
\hline $\begin{array}{c}\text { LC doped with } \\
\text { PANI+C } 60\end{array}$ & 1 & 0.3 & 20 & $1.1 \times 10^{-3}$ & current \\
\hline $\begin{array}{c}\text { LC doped with } \\
\text { PANI+C } 60\end{array}$ & 6 & 0.3 & 20 & $1.3 \times 10^{-3}$ & current \\
\hline Source: Author
\end{tabular}

It should be remarked once again, that the change of the refractive index has been estimated under the Raman-Nath diffraction conditions. The following equation has been made (Akhmanov, 1997), which firstly has been applied for the calculations for the structured organic media in paper (Kamanina, 2005):

$$
\eta=\frac{I_{1}}{I_{0}}=\left(\frac{\pi \Delta n_{i} \mathrm{~d}}{2 \lambda}\right)^{2}
$$

where $\Delta n_{\mathrm{i}}$-induced change of the refractive index, $I_{1}$ is the intensity in the first diffraction order, $I_{0}$ is the input laser intensity, $d$ is the thickness of the medium, $\lambda$ is the wavelength of the light incident on the medium. In the current experiment the wavelength of the laser beam was $532 \mathrm{~nm}$, as the second harmonic of the pulsed Nd-laser, and the pulse width $\tau$ was close to $20 \mathrm{~ns}$.

\section{Conclusion}

To summarize the obtained results and proposed consideration, one can testify towards the following aspects. Different physical mechanisms can be considered in order to explain the OL effect. Among them, indeed, the reverse saturable absorption is the main mechanism, which is responsible for the OL process in the visible spectral range. Under the sensibilization model matrix conditions the bathochromic shift can be found, thus, the intermolecular complex formation can be used too as the OL mechanism, which permits to extend the spectral range up to IR one. Moreover, the energy losses via diffraction from the nanostructured organics can extend the number of the OL mechanism efficiently. As the novel additional mechanism, shown in the current paper, the energy losses via reflection from the surfaces can be considered under the condition when the material body has been sensitized with the varied content of the nanoobjects. The core of the nanoobjects influences on the surface relief of the materials dramatically, which takes part in the attenuation of the laser beam. As the more perspective nanoobjects, which provoke the surface relief change, namely the CNTs can be used. Based on the data presented one can conclude that the area of the OL process application can be extended and supported by a different model of the OL mechanism.

\section{Acknowledges}

Author would like to thank her colleagues from the Lab for Photophysics of media with the nanoobjects (Vavilov State Optical Institute, Saint-Petersburg, Russia) for the helpful discussion at the Lab seminars. Moreover, some part of the obtained results has been discussed at the seminar in Vincha 
Institute (Belgrade, Serbia, 2017), in Nuclear Research Institute (Gatchina, Saint-Petersburg, Russia, 2017) and at Prague CBU Research Institute (Prague, Czech Republic, 2019).

\section{References}

Akhmanov, S.A. and Nikitin, S.Yu. Physical Optics. Oxford, 1997, 504 p. ISBN 0-19-851795-5

Belousov, V.P., Belousiva, I.M., Budtov, V.P., Danilov, V.V., Danilov, O.B., Kalintcev, A.G., Mak, A.A. (1997). Structured, physical-chemical and nonlinear optical properties of the fullerenes. Optical Journal, 64(12), 3-37.

Brabec, C. J., Padinger, F., Sariciftci, N. S., Hummelen, J. C. (1999). Photovoltaic properties of conjugated polymer/methanofullerene composites embedded in a polystyrene matrix. Journal of Applied Physics, 85(9), 6866-6872.

Couris, S., Koudoumas, E., Ruth, A. A., Leach, S. (1995). Concentration and wavelength dependence of the effective thirdorder susceptibility and optical limiting of $\mathrm{C}_{60}$ in toluene solution. J. Phys. B: At. Mol. Opt. Phys., 8, 4537-4554.

Hosoda, K., Tada, R., Ishikawa, M. and Yoshino, K. (1997). Effect of $\mathrm{C}_{60}$ doping on electrical and optical properties of poly[(disilanylene)oligophenylenes]. Jpn. J. Appl. Phys., Part 2, 36(3B), L372-L375.

Kamanina, N.V. (2001). Peculiarities of optical limiting effect in $\pi$-conjugated organic systems based on 2-cyclooctylamino5-nitropyridinedoped with $\mathrm{C}_{70}$. Journal of Optics A: Pure and Applied Optics, 3(5), 321-325.

Kamanina, N.V. (2002). Mechanisms of optical limiting in $\pi$-conjugated organic system: fullerene-doped polyimide. Synthetic Metals, 127(1-3), 121-128

Kamanina, N.V. (2005). Fullerene-dispersed liquid crystal structure: dynamic characteristics and self-organization processes. Physics-Uspekhi, 48(4), 419-427.

Kamanina, N.V. (2019). Liquid crystal materials orientation using new approach. Proceed. of CBU International conference on innovations in science and education, March 20-22, 2019, PRAGUE, CZECH REPUBLIC, 2019, 933-937, DOI: 10.12955/cbup.v7.147. WWW.JOURNALS.CZ.

Belousov, V.P., Belousiva, I.M., Gavronskaya, E.A., Grigor'ev V.A., Danilov, O.B., Kalintsev, A.G., Krasnopolskij V.E., Smirnov, V.A., Sosnov E.N. (1999). About optical limiting mechanisms in the fullerene-containing media. Optics and Spectroscopy, 87(5), 845-852.

Belousova, I.M., Belousov, V.P., Danilov, O.B., Grigor'ev, V.A., Kalintsev, A.G., Zgonnik, V.N., Kamanina, N.V., Zhevlakov, A.P., Kris'ko, A.V., Mironova, N.G., Sosnov, E.N., Gavronskaya, E.A., Smirnov, V.A., Yur'ev, M.S., Ponomarev, A.N., Yashin, V.E. (2001). Photodynamics of nonlinear fullerene-containing media, Proceed. SPIE, 4353, 75-83. Kamanina, N.V., Kuzhakov, P.V., Serov, S.V., Kukharchik, A.A., Petlitsyn, A.A., Barinov, O.V., Borkovskii, M.F., Kozhevnikov, N.M., Kajzar, F. (2013). Nanostructured materials and their optical features. Proc.SPIE, 8622, 86221B-9. DOI:10.1117/12.979822.

Kamanina, N.V., Serov, S.V., Shurpo, N.A., Likhomanova, S.V., Timonin, D.N., Kuzhakov, P.V., Rozhkova, N.N., Kityk, I.V., Plucinski, K.J., Uskokovic, D.P. (2012). Polyimide-fullerene nanostructured materials for nonlinear optics and solar energy applications. J Mater Sci: Mater Electron, 23(8), 1538-1542. DOI 10.1007/s10854-012-0625-9.

Kamanina, N.V. and Uskokovic, D.P. (2008). Refractive Index of Organic Systems Doped with Nano-Objects, Materials and Manufacturing Processes, 23, 552-556.

Kamanina, N.V. and Vasilenko, N.A. (1995). High-speed SLM with a photosensitive polymer layer, Electron. Lett. 31(5), 394-395. DOI: 10.1049/el:19950258.

Kamanina, N.V., and Vasilenko, N.A. (1997). Influence of operating conditions and of interface properties on dynamic characteristics of liquid-crystal spatial light modulators. Opt. Quantum Electron., 29(1), 1-9. DOI:10.1023/A:1018506528934 Published: JAN 1997.

Krätschmer, W., Fostiropoulos, K., Huffman, D.R. (1990).The infrared and ultraviolet absorption spectra of laboratoryproduced carbon dust: evidence for the presence of the $\mathrm{C}_{60}$ molecule. Chem. Phys. Lett., 170(2-3), 167-170.

Krätschmer, W., Lamb, L.D., Fostiropoulos, K., Huffman, D.R. (1990). Solid C60: a new form of carbon. Nature, 347, 354358

Khoo, I.C., Le, H., Liang, Y. (1994). Observation of orientational photorefractive effect in nematic liquid crystals. Optics Letters, 19(21), 1723-1725.

Liu, Huimin, Taheri, B., Weiyi, Jia. (1994).Anomalous optical response of $\mathrm{C}_{60}$ and $\mathrm{C}_{70}$ in toluene. Phys. Rev. B, 49(15), 10166-10169.

Namilae, S., Chandra, N., Shet, C. (2004). Mechanical behavior of functionalized nanotubes. Chemical Physics Letters, 387 , 247-252.

Ould-Moussa, N., Blanc, Ch., Zamora-Ledezma, C., Lavrentovich, O.D., Smalyukh, I.I., Islam, M.F., Yodh, A.G., Maugey, M., Poulin, P., Anglaret E., Maurizio N. (2013). Dispersion and orientation of single-walled carbon nanotubes in a chromonic liquid crystal. Liquid Crystals, 40(12), 1628-1635, DOI:10.1080/02678292.2013.772254.

Robertson, J. (2004). Realistic applications of CNTs. Materials Today. 7, 46-52.

Sapurina, I., Mokeev, M., Lavrentev, V., Zgonnik, V., Trcova, M., Hlavata, D., Stejskal, J. (2000). Polyaniline complex with fullerene $\mathrm{C}_{60}$. European Polymer Journal, 36(11), 2321-2326. 